\title{
Thoughts on 2020 World Pest Day
}

\author{
Xiaoyun Huang ${ }^{1}$
}

In view of the "Big Harms" that may be attributable to the "Small Pests" around us, the launch ceremony of "World Pest Day" was held at Beijing Hotel on June 6, 2017. The original goals and development processes of World Pest Day are to gain well-deserved respect for the global pest control industry and to open up a new way of thinking to facilitate industry's public-facing platform and ultimately to achieve positive changes in public health. Since the establishment of World Pest Day, different countries and regions have carried out various activities based on specific needs of the countries or regions. This paper also seeks to appeal to the general public about the great importance of pest control industry on public health and to emphasize the key role pest control industry plays on the prevention and control of infectious diseases, especially during the period of the coronavirus disease 2019 (COVID-19) pandemic in 2020.

Preparation, Gestation, Planning, Action and Result of World Pest Day (2015-2017): Based on Global Vector Control Response 2017-2030, 80\% of the world's population is at risk of one or more vectorborne diseases (VBDs) and over 700,000 deaths are caused by VBDs annually. This poses a clear and present danger to public health.

In order to raise the public's awareness about the prevention and control of VBDs, I first proposed in May 2015 the idea of designating June 6 as "World Pest Day". With the strong support and guidance of Dr. Jianguo $\mathrm{Xu}$, President for the Chinese Pest Control Association (CPCA), and the joint support of Qiyong Liu, the head of the World Health Organization (WHO) Collaborating Centre for Vector Surveillance and Management (WHOCCVSM), in 2015 and 2016, the CPCA began to carry out research on various internationally-recognized awareness days, drafted the World Pest Day Proposal, and sent staff to various international conferences to promote the initiative and to solicit signatures from international colleagues. Nearly 10,000 representatives from more than 30 national associations around the world and the WHO became signatories to support "World Pest Day" including Nobel laureate Tu Youyou who signed on to lend her support. With the efforts of Dr. Xu Jianguo, who is also a member of academicians of the Chinese Academy of Engineering, World Pest Day received strong support from $\mathrm{Mr}$. Zhong Nanshan and other 41 Chinese academicians as well as 21 members of the National Committee of the Chinese People's Political Consultative Conference. These efforts paved the way for the official launch of World Pest Day (1).

On June 6, 2017, more than 300 delegates, including pest control industry leaders, scientific research elites, celebrities, and media members from more than 20 countries and regions on 5 continents, witnessed the birth of "World Pest Day" (2). Led by the CPCA in collaboration with the WHOCCVSM, FAOPMA, National Pest Management Association (NPMA) in the United States, Confederation of European Pest Control Associations (CEPA) and other co-sponsors, "World Pest Day" as born (3). More than 200 Chinese and foreign media members participated in the event. Associations and other organizations from the United States, Australia, India, Britain, France, Spain, Italy, Germany, Hungary, Peru, South Africa, Mexico, and other countries and regions have held press conferences and events (4).

Theme, Purpose and Activities of World Pest Day (2018-2019): Pests pose a serious threat to the food security of China and the world. China's total annual grain production's loss caused by pests which accounts for nearly $30 \%$ of the total output loss. Each year, food losses caused by pest damage amount to 1.5 to 6 million tons, and direct economic losses exceed 2 billion yuan. From 2006 to 2015, pests that are commonly found in grain storage facilities such as Sitophilus oryzae, Tribolium castaneum, Rhizopertha dominica, and Tineidae caused losses of 301,900 tons of soybeans, 3.336 million tons of wheat, and 4.988 million tons of rice in China.

On June 6, 2018, the theme of the World Pest Day was "Controlling Pests and Ensuring Food Safety." The main venue of World Pest Day was in Lisbon, Portugal. Representatives from more than 80 countries participated in the event. Dr. Qiyong Liu, the chief scientist for vector control of China CDC and the head 
of the WHOCCVSM, delivered a keynote speech titled "Pest Control for Public Health and Food Safety". Also on behalf of China, I was awarded the "Global Industry Ambassador" by NPMA and the first ever "Outstanding Contribution Award" awarded by FAOPMA.

Additionally, events in Asia, Oceania, Europe, America, and Africa took place on the same day. With the theme "Controlling Pests and Ensuring Food Safety", the State Key Laboratory of Infectious Disease Prevention and Control (SKLID) opened its lab to the general public in Beijing, China. Dr. Jianguo Xu gave a keynote speech on "Controlling Pests and Ensuring Food Safety". There were expert forums, pest prevention and control science education sessions, pest control live demonstrations, and interactive sessions with the media. A series of key laboratory opening activities were organized, such as the popular science exhibition on the subject of pest ecology, its harms, identification of pests, exhibition of history of infectious diseases control, and the public viewing of laboratory experiments. Education sessions were held with easy to understand analysis about the damages caused by pests from the cultivation and growing of raw food materials, to harvesting and storage, to transportation, processing, and consumption. Comprehensive prevention and control measures on effective pest control were also presented. During the week of June 6, various events took place in more than 30 cities, including Beijing, Shanghai, Tianjin, Chengdu and Chongqing in China. More than 80 Chinese media members reported the event, and World Pest Day posters were displayed anywhere around a number of cities. Each community posted information on World Pest Day on bulletin boards with more than 18 million people viewing the information nationwide every day (5).

On June 6, 2019, the theme of World Pest Day was "Pest Control for Healthy Communities". Communities are the fabric of society, and building healthy communities is an important part of achieving a healthy China. With the increasing level of people's awareness on public health, pest control in our community has become one of the vital aspects of building a healthy community. "2019 World Pest Day Live Meeting" with the theme "Pest Control for Healthy Communities" was jointly hosted by the CPCA and Tianjin Pest Control Association in Tianjin Municipality, China (G). Experts were invited to give scientific lectures on "Pest Control for Healthy Communities"; the public announcement cartoon short film "Fighting against Mosquito" also premiered; expert consultation on pest control technology, live demonstration of pest control project, interactive media sessions were carried out; a series of activities were organized such as the scientific exhibition on pest control and various forms of science education about community pest control. Around June 6, 2019, more than 40 cities in China including Shanghai, Guangzhou, Shenzhen, Chongqing, Chengdu, Hefei, Nanjing, and Fuzhou, organized World Pest Dayrelated activities for the general public, which was widely reported by more than 120 Chinese media outlets (7).

Theme, Prospect and Suggestions of the 2020 World Pest Day: Rodent-related threats in China are particularly destructive, causing annual agricultural losses of about 15 million tons and economic losses of tens of billions RMB. At the same time, rodent-borne diseases also pose a serious threat to public health. Among the dozens of rodent-borne diseases, plague, hemorrhagic fever with renal syndrome (HFRS), and leptospirosis are the most threatening ones. Among them, HFRS is the most common disease with an annual case count of more than 100,000 in China in the past, but had been limited to between thousands of cases to tens of thousands of cases in recent years due to effective rodent control. Presently, with rapid economic development, changes in ecology, climate, and land use patterns, as well as the development of tourism, the number and density of rodents are gradually increasing, which has exacerbated the high incidence of emerging rodent-borne diseases. It not only poses a serious threat to people's health but also has a potential negative impact on social stability and economic development.

To this end, the theme of World Pest Day 2020 in China will be Rodent Control for Health. In 2020, the sudden outbreak of COVID-19 disrupted the daily routine of the entire world, which has had a serious impact on our work, lives, and the economy. In the global fight against the pandemic, the pest control industry from all countries and regions are also facing severe impacts and challenges. Many restaurants, hotels, factories, etc. have chosen to forego pest control services for economic reasons, which has led to the suspension of routine pest control activities and could increase the outbreak risks of VBDs such as dengue and plague. The COVID-19 pandemic will inevitably exacerbate public health crises in many countries and strain public health resources. As a former president of the FAOPMA, I appeal to the public health authorities 
of all countries and regions, pest control professionals and general public: It is time to recognize the enormous contribution of pest control industry made to public health and to understand the key role pest control industry plays in the prevention and control of infectious diseases.

doi: $10.46234 / \mathrm{ccdcw} 2020.113$

\footnotetext{
${ }^{1}$ The Former President of the Federation of Asian \& Oceania Pest Managers Associations, Vice President and Secretary-general of the Chinese Pest Control Association.
}

Submitted: May 12, 2020; Accepted: May 20, 2020

\section{REFERENCES}

1. Du J. "World Pest Day" conference held in Beijing on June 6th, Tu Youyou and Zhong Nanshan support it. China Daily.com. 2017-06-07.
http://cnews.chinadaily.com.cn/baiduMip/2017-06/07/cd_29653663. html. [2020-05-10]. (In Chinese)

2. Hu SZ. The launch event for the world pest day held in Beijing, China on June 6. Huanqiu.com. 2017-06-06. https://china.huanqiu.com/ article/9CaKrnK3fGd. [2020-05-10]. (In Chinese)

3. Shi N. World pest day-WHO representative Fabio scano delivered a speech. Tecent. 2017-06-13. https://v.qq.com/x/page/n0513kewvmt. html. [2020-5-10].

4. Qiu LY. The rise of public spiritedness of Chinese pest control industry. Chin J Hyg Insect Equip 2018;24(3):310 - 2. http://dx.doi.org/10. 19821/j.1671-2781.2018.03.029. (In Chinese).

5. Sun H. 2018 world pest day: Controlling pest and ensuring food safety. Xinhua Net. 2018-06-07. http://www.xinhuanet.com/health/2018-06/ 07/c_1122951365.htm. [2020-05-10]. (In Chinese).

6. Chen QB. 2019 "World Pest Day" public welfare event held in Tianjin to control pests and build a healthy community. CNR News. 2019-0606. http://www.cnr.cn/tj/jrtj/20190606/t20190606_524641983.shtml. [2020-05-10]. (In Chinese).

7. Chen J. World pest day propose to build healthy communities. Health Newspaper. 2019-06-07. http://szb.jkb.com.cn/jkbpaper/html/2019-06/ 07/content_249295.htm. [2020-05-10]. (In Chinese). 